\title{
A utilização de boletim eletrônico no setor de referência:um estudo de caso
}

Robson da Silva Teixeira

\begin{abstract}
Bibliotecário-chefe da Biblioteca do Instituto de Física da Universidade Federal do Rio de Janeiro (UFRJ).Mestre em Desenvolvimento Local (UNISUAM)
\end{abstract}

http://dx.doi.org/10.1590/1981-5344/1731

Este artigo trata da elaboração de um boletim eletrônico como ferramenta de enfrentamento aos desafios impostos pelas Novas Tecnologias da Informação e Comunicação na disseminação da informação nas Universidades Públicas Brasileiras, tendo como parâmetro um estudo de caso no setor de referência da Biblioteca Plínio Sussekind Rocha do Instituto de Física da Universidade Federal do Rio de Janeiro (IF/UFRJ).

Palavras-chave: Bibliotecas - Serviço de Referência; Boletim eletrônico; Boletim informativo.

\section{The use of electronic industry bulletin} reference

This article deals with the development of a electronic newsletter as a tool for coping with the challenges posed by New Technologies of Information and Communication Technologies in the dissemination of information on Brazilian Public Universities, with the parameter a case study in the industry reference library Pliny Sussekind Rock Physics Institute of the Federal University of Rio de Janeiro (IF / UFRJ).

Keywords: Libraries - Reference Service; Electronic Bulletin; Newsletter.

Recebido em 19.12.2013 Aceito em 07.05.2015 


\section{Introdução}

Há um enorme campo de trabalho para o serviço de referência em bibliotecas universitárias - e esse trabalho é urgente. Evidentemente, competem às universidades, produtoras e difusoras do conhecimento, zelar por sua documentação histórica e principalmente pela excelência na prestação de serviços e produtos aos seus usuários. É fundamental conscientizar-se para a importância da qualidade no serviço de referência em bibliotecas universitárias, já que esse serviço é imprescindível para um correto atendimento aos usuários.

O objetivo deste artigo esta baseado numa análise mais ampla do serviço de referência e informação, permitindo vislumbrar o todo do setor de Referência, com seus serviços direcionados não só para o atendimento e interação direta com o usuário, mas também por meio de atividades que antecipem a demanda de informações, orientem o usuário na utilização dos recursos e dissemine os recursos de informação disponíveis, objetivo principal do boletim eletrônico da Biblioteca do Instituto de Física. Garcez e Rados (2002) destacam que é papel das bibliotecas oferecerem serviços e produtos especializados agregando valor aos mesmos, com criatividade em sua realização e formato sem perder o foco nos usuários e na satisfação de sua necessidade de informação.

A idéia de desenvolver um boletim eletrônico surgiu a partir do questionamento do pesquisador sobre a real capacidade do serviço de referência de uma Biblioteca Universitária em atender de forma satisfatória as necessidades dos seus usuários, a partir deste questionamento, e da constatação de que a Biblioteca necessitava de um canal mais direto, dinâmico e atual para disseminação da informação, surgiu à necessidade de desenvolver o Boletim Eletrônico da Biblioteca do Instituto de Física (BIF).

O Boletim Eletrônico da BIF é uma iniciativa dos profissionais da informação da Biblioteca Plínio Sussekind Rocha do Instituto de Física da Universidade Federal do Rio de Janeiro (IF/UFRJ) para enfrentar os desafios impostos pelas Tecnologias da Informação e Comunicação, que mudou completamente a forma das Bibliotecas oferecerem produtos e serviços aos seus usuários, fazendo com que elas tenham que reavaliar suas funções e objetivos nesta Nova Era do Conhecimento. Fato constatado por Figueiredo (1991) quando ele afirma que uma biblioteca é mensurada pela eficácia do serviço de referência, desta forma, os serviços e produtos de uma biblioteca devem ter como meta atender às necessidades do usuário de modo eficiente, ou seja, cabe ao bibliotecário de referência usar de técnicas variadas para otimizar a prestação de serviços.

As atividades de um setor de referência representam uma ampla gama de ação desde o tradicional serviço de referência até a moderna busca de dados on-line. Marcondes, Mendonça e Carvalho (2006) observam que esses podem ser considerados como uma evolução dos serviços biblioteconômicos via Internet. Garcez e Rados (2002) alertam 
que as bibliotecas devem integrar os recursos presentes no mundo impresso e no mundo digital. Assim, a biblioteca não só interage com o meio ambiente interno, mas também com ambiente externo, de forma sempre dinâmica, pesquisando, disseminando e alimentando o conhecimento gerado no meio acadêmico (CARVALHO; LUCAS, 2001).

O Boletim Eletrônico da Biblioteca do Instituto de Física é uma ferramenta que oferece aos seus leitores, informações sobre o uso de produtos e serviços da Biblioteca, tais como: livros eletrônicos, variedades e novidades do acervo, dentro outros, fazendo com que a Biblioteca alcance o seu objetivo de contribuir para a inovação e para o crescimento do ensino e pesquisa em Física no Brasil, em especial, no Instituto de Física da Universidade Federal do Rio de Janeiro (IF/UFRJ).

\section{Instituto de Física e sua história}

O Instituto de Física da Universidade Federal do Rio de Janeiro (IF/UFRJ) foi criado em 19 de março de 1964 e faz parte do Centro de Ciências Matemáticas e da Natureza (CCMN) da referida universidade. Antes da criação do Instituto, o curso de física fazia parte da Faculdade Nacional de Filosofia (FNFI) e reunia cinco cátedras de Física, com vistas a sua finalidade de formar Bacharéis e Professores Licenciados. O Instituto de Física (IF) foi criado por ocasião da reforma universitária, que reuniu os cursos de física então existentes em escolas e faculdades do Rio de Janeiro pertencentes à Universidade do Brasil (UB). O IF buscava constituir-se com a contratação de professores necessários às atividades de ensino, e procurava quadros superiores para a implantação de atividades de pesquisa e a preparação para a pós-graduação, que, até então, não existia no Instituto ${ }^{1}$.

\section{Ambiente da pesquisa: breve histórico}

Segundo Brandão e Carvalho (2009) as Bibliotecas obedecem às normas estabelecidas pelo Sistema de Bibliotecas da Universidade Federal do Rio de Janeiro (SIBI/UFRJ), que tem o objetivo de dar apoio aos programas de ensino, pesquisa e extensão, desenvolvendo-se de acordo com o planejamento da Instituição e formando o seu acervo em consonância com as emendas das diferentes disciplinas oferecidas, em nível de graduação e de pós-graduação. O SIBI disponibiliza o acervo e serviços das Bibliotecas através do sistema Aleph/Minerva, desenvolvido para tratamento, armazenamento e recuperação de informações bibliográficas e multimídia.

A Biblioteca Plínio Sussekind Rocha, vinculada ao Instituto de Física, tem um acervo de aproximadamente 12.000 livros e 250 títulos de periódicos (nacionais e estrangeiros). Num primeiro momento, a Biblioteca do Instituto de Física disponibilizou o seu acervo bibliográfico por meio da

\footnotetext{
1 UFRJ história. Disponível em: <http://www.ufrj.br/pr/conteudo_pr.php?sigla=HISTORIA>. Acesso em: 13 mar. 2012.
} 
base de dados ALEPH, o que provocou uma mudança significativa em seus serviços, uma vez que a consulta ao catálogo foi automatizada. Percebeuse, desde então, a necessidade de automatizar o serviço de empréstimo que era realizado de forma manual e que não condizia com a realidade da Universidade e acarretava vários problemas, dentre eles, o tempo na realização dos empréstimos, falta de agilidade na reserva e controle de cobrança aos usuários em atraso. Entretanto, diante dos percalços, como o déficit de pessoal, a biblioteca somente em 2008, com o apoio da Bibliotecária do Núcleo de Computação Eletrônica da UFRJ (UFRJ/NCE), conseguiu realizar a automação do setor de circulação. Atualmente, a Biblioteca do IF está completamente automatizada, e oferece vários serviços e produtos aos seus usuários, tais como: Renovação e Reserva Online, Espaço Conexão (espaço destinado à pesquisa ao Portal Capes, livros Eletrônicos, Bases de Dados e o novo site da Biblioteca) e o Setor de Multimeios (divulgação de Cds/Dvds de livros e Teses/Dissertações), porém não existia, até então, um canal que reuni-se e dê-se acesso, num mesmo recurso de informação, essa gama de produtos e serviços.

\section{Revisão de literatura}

Em se tratando de uma pesquisa voltada para uma biblioteca universitária, convém definir biblioteca universitária, serviço de referência e Boletim Informativo.

Como parte integrante da Universidade, a biblioteca universitária pode ser definida, conforme Silveira (1992), como uma biblioteca dedicada ao fornecimento de suportes informacionais as instituições de ensino superior para que elas possam desempenhar suas atividades de ensino, pesquisa e extensão universitária.

A biblioteca universitária inserida no contexto acadêmico necessita estar de acordo com a finalidade da Instituição de Ensino Superior (IES), pois Cunha (2010) acredita que as bibliotecas universitárias são organizações complexas, com múltiplas funções e uma série de procedimentos, produtos e serviços que foram desenvolvidos ao longo de décadas. No entanto, o seu propósito fundamental permaneceu o mesmo, isto é: proporcionar acesso ao conhecimento. Esse acesso ao conhecimento é que irá permitir que o estudante, o professor e o pesquisador possam realizar suas aprendizagens ao longo da vida. Segundo Tarapanoff, Klaes e Cormier (1996), a universidade é uma organização social, que interage com o meio ambiente interno e externo, de forma dinâmica, no tempo e no espaço. Para Cunha (2010) a biblioteca universitária existe porque presta serviços de atendimento no campus, de forma presencial ou virtual. O atendimento presencial tenderá a decrescer à medida que os usuários passem a utilizar, de forma intensa e variada, as inúmeras ferramentas disponíveis na chamada Web 2.0.

Para compreender as características do serviço de referência e informação de uma Biblioteca Universitária e apresentar suas especificidades, é necessário também conceituar, de maneira clara, as 
linhas básicas de atuação deste tipo de serviço em seu sentido tradicional e em seus pontos teóricos. Para tanto, serão utilizadas algumas conceituações e caracterizações desenvolvidas por alguns especialistas no assunto.

Macedo (1990) conceitua o serviço de referência em dois sentidos. Em sentido restrito e em sentido amplo. No primeiro caso, diz que:

A essência do conceito de Referência é o atendimento pessoal do bibliotecário, profissional preparado para este fim [...] ao usuário que, em momento determinado, o procura para obter uma publicação ou informação por ter alguma dificuldade ou para usar a biblioteca e seus recursos e precisa de orientação; ou ainda, não encontrando a informação na biblioteca, precisa ser encaminhado para outra instituição [...].

Em sentido amplo, segundo a autora, para compreender o serviço de referência como um todo, é preciso vê-lo em duas facetas. A primeira conceitua o serviço de referência como sendo a interface direta entre informação e usuário, através do bibliotecário de referência, que responde as questões e auxilia, com seus conhecimentos profissionais, os usuários. A segunda faceta, chamada de serviço de referência e informação um recorte do todo da biblioteca, com pessoal, arquivo, equipamento, método próprio para melhor canalizar o fluxo final da informação e melhorar o seu uso, e meio de linhas de atividades (MACEDO, 1990).

Figueiredo (1996), afirma que:

As tendências futuras, realmente apontam para o serviço de referência e informação on line sugerindo cada vez mais a utilização de recursos tecnológicos para a disseminação de informações as bases de dados computadorizadas, que já estão ocasionando o cancelamento de assinaturas de periódico, substituídas por base de dados on line, pagas conforme a freqüência e o uso, e a implementação de vídeo texto e tele texto, o oferecimento de disseminação seletiva da informação online.

Porém, para que a geração do conhecimento seja cíclica, e a realidade citada por Figueiredo seja efetiva, é necessário prover o acesso, dinamizar, sociabilizar e divulgar esta produção, bem como veicular meios de pesquisa que promovam e facilitem o acesso a informações as diversas áreas do conhecimento humano, e o boletim eletrônico pode ser um canal eficaz, principalmente num contexto extremamente dinâmico, como a Internet, onde a plataforma Web é considerada um dos suportes mais utilizados e crescentes que dinamiza, sociabiliza e inova informações, ao usuário onde quer que esteja.

Somado a isso, Lynch (2001), diz que: 
Agora que estamos começando a ver, nas bibliotecas, o texto completo aparecendo online, acho que muito em breve estaremos prestes a cruzar uma espécie de limite crítico, onde as publicações que não estão imediatamente disponíveis em texto integral serão uma espécie taxada como de segunda classe, não porque a qualidade seja baixa, mas apenas porque as pessoas vão preferir a acessibilidade das coisas que podem ser disponibilizadas imediatamente. Elas vão se tornar muito menos visíveis para a comunidade dos leitores.

Segundo Cunha (2010) o serviço de referência nas bibliotecas, se tornou o principal ponto de contato para os estudiosos pedirem ajuda no exercício da sua pesquisa entre os acervos da biblioteca. Reforçando esta afirmação temos Grogan (2001) que diz:

[...] a proporção crescente das fontes de informação tradicionais do bibliotecário de referencia que se apresentam na forma de base de dados informatizadas, ao lado de inúmeras ferramentas novíssimas, disponíveis para buscas numa variedade de novas formas, representa um progresso do serviço de referência.

O boletim eletrônico ( $\mathrm{BE}$ ), também conhecido como boletim informativo, segundo a Wikipédia (2015), pode ser definido como um tipo de publicação de distribuição regular a assinantes e que aborda geralmente um determinado assunto. Generalizam-se cada vez mais os boletins informativos distribuídos como mensagem eletrônica que o usuário pode receber via Internet após efetuar um cadastramento em algum site. Os Boletins informativos/Eletrônicos, são geralmente enviados por correio electrônico mas também podem ser enviadas por SMS, MMS ou outros tipos de comunicação electrônica. Algumas empresas os usam para fornecer novidades e informações.

\section{Boletim eletrônico: um olhar diferenciado para o serviço de referência}

Segundo Lima (2012) as Tecnologias de Comunicação e Informação influenciam as transformações e inovações nos processos de difusão da informação. Exemplos disso podem ser verificados, especialmente, em relação ao ambiente da Internet e da $W e b$, onde as mudanças estão cada vez mais rápidas.

Trabalhos recentes (CARLSON, 2002; LAMB, 2005) têm revelado que os comportamentos e hábitos de busca informacional estão em constante mudança. A Internet está cada vez mais onipresente e continua crescendo ainda mais pela introdução de novos e melhores algoritmos nos mecanismos de busca. A World Wide Web (web) se tornou o maior 
depósito de informação do mundo. Em meados de 2010, um bilhão e oitocentos milhões de pessoas - 26,6 \% da atual população mundial passaram a usar a internet (INTERNET WORLD STATS, 2010).

A velocidade das transformações que estão ocorrendo no processamento e uso da informação tem obrigado as instituições a reverem suas práticas, por isso as tecnologias de interação e colaboração estão sendo vistas como nova modalidade de construção coletiva de conhecimento.

Nessa perspectiva as instituições de ensino superior de um modo geral têm buscado criar e adequar produtos e serviços ao novo contexto, representado pelo crescimento do conhecimento $e$ a conseqüente explosão bibliográfica disponibilizada através da internet, gerando uma necessidade de uso intensivo das novas tecnologias.

O boletim eletrônico foi elaborado com o objetivo de fornecer informação para os leitores acerca das principais atividades, produtos e serviços da Biblioteca. O diferencial do Boletim Eletrônico do IF é agregar, além das informações já disseminadas pela Biblioteca, outras informações que também são de interesse da comunidade acadêmica, como por exemplo, notícias, divulgação científica, variedades e o esclarecimento de dúvidas observadas no contato com o usuário. Utilizou-se como parâmetro a $4^{a}$ Lei de Ranganathan: "Poupe o tempo do usuário", ao criar um produto para disseminação da informação e enviá-lo diretamente para o e-mail dos usuários, atribuindo dinamismo, atualidade e objetividade ao produto. Carvalho e Lucas (2001, p. 2) reforçam esta afirmação quando dizem que o serviço de referência deve ter como base:

[...] prover o acesso, dinamizar, sociabilizar e divulgar esta produção, bem como veicular meios de pesquisa que promovam e facilitem o acesso a informações as diversas áreas do conhecimento humano $[\ldots]$.

Acredita-se que dentro desse universo essencialmente dinâmico, que a plataforma Web pode ser considerada como um dos suportes mais utilizados, pois dinamiza, sociabiliza e atualiza informações ao usuário, onde quer que ele esteja.

Pierre Lévy é um dos grandes defensores das novas tecnologias da informação e do ciberespaço, enquanto instrumento de ampliação do conhecimento humano; pois entende a Internet como um espaço interativo, e importante ferramenta de colaboração. Blattmann e Silva (2007) esclarecem que a evolução e desenvolvimento da Web 2.0 culminaram em diferentes ferramentas que propiciam a partilha interativa de saberes, através dos softwares sociais. 


\section{A elaboração do boletim eletrônico}

Para cumprir os objetivos traçados, a equipe de profissionais da informação desenvolveu o projeto em etapas, que serão descritas a seguir:

1. Foi desenvolvido um logo personalizado para o $B E$, para que ele tivesse uma identificação imediata com o seu público;

2. O BE foi dividido por seções e cada uma delas oferecendo um serviço diferencial, tais como:

Seção 1: Fique por dentro: esta seção é dedicada a orientar os usuários da Biblioteca com informação e dicas que podem sanar dúvidas e resolver problemas com mais rapidez.

Seção 2: Já existe... e você não sabia ! : trata de serviços e/ ou produtos que a Biblioteca já oferece aos seus usuários, mas que muitos desconhecem.

Seção 3: Livros eletrônicos: esta seção dá acesso à base de livros eletrônicos da Springer Link publicados no período de 2005-2009, em várias áreas do conhecimento, além de selecionar itens de relevância para área da Física.

Seção 4: Versões eletrônicas de livros clássicos: seção focada no projeto Gutemberg, que se destina a digitalizar e disponibilizar, gratuitamente, livros de domínio público, tornando-se uma excelente fonte de pesquisa para quem precisa de livros clássicos e de importância histórica e social.

Seção 5: Novas aquisições da Biblioteca: destina-se a informar aos leitores as mais recentes aquisições da Biblioteca.

Seção 6: Novas Teses \& Dissertações: a Biblioteca do IF é depositária da versão impressa e online de todas as Teses e Dissertações produzidas pelos discentes dos programas de Pós-graduação do IF; esta seção disponibiliza a referência e o link para acesso online.

Seção 7: 0 IF Divulga: esta seção destina-se a divulgar informações científicas, com foco na novidade, tanto no conteúdo quanto no formato, por exemplo, a tese do professor Plínio Sussekind Rocha (patrono da Biblioteca) digitalizada e disponibilizada na Minerva (catálogo online). Seção focada em informação de diversas áreas, que podem ou não estar relacionadas à Física, com o objetivo de oferecer uma cultura geral aos leitores. 
Seção 8: Notícias; tem por objetivo trazer à luz, questões que estão sendo discutidas dentro da área da Física, divulgadas nos mais diversos meio de comunicação, como por exemplo, jornais e revistas eletrônicas, sites de notícias e portais online de Física.

Seção 9: Sustenta o quê?: esta seção trata de iniciativas que estão sendo desenvolvidas para o enfrentamento aos desafios impostos pelo uso irresponsável dos recursos naturais e os danos causados ao meio ambiente. Essa seção representa uma pequena iniciativa de trazer a público algumas iniciativas, bem sucedidas, de atividade com forte cunho social e fundamental preocupação com a questão da sustentabilidade e a preservação da natureza. Ressalta que as matérias aqui publicadas podem ou não estar relacionadas à Física.

\section{Considerações finais}

Os objetivos fixados para este artigo não permitem que o assunto serviço de referência em bibliotecas universitárias seja tratado com a profundidade que merece. Ele é por demais amplo. Pesquisou-se o serviço de referência numa biblioteca universitária com objetivo de desenvolver um boletim eletrônico que divulgue o Serviço de Referência da Biblioteca do Instituto de Física da Universidade Federal do Rio de Janeiro (IF/UFRJ), destacando os principais serviços e produtos da Biblioteca e assim contribuir para que a comunidade acadêmica cumpra com seus compromissos educacionais e tenham como aliados nos momentos mais difíceis, o profissional da informação.

O objetivo principal deste artigo - constitui um boletim eletrônico que divulgue o Serviço de Referência da Biblioteca do Instituto de Física da Universidade Federal do Rio de Janeiro (IF/UFRJ) - foi alcançado. O Boletim Eletrônico já está disponível aos usuários na página da Biblioteca na Internet (WWW.biblioteca.if.ufri.br). Atualmente, o cadastro de usuários para recebimento do boletim eletrônico por e-mail tem 638 usuários cadastrados, porém este número cresce, a todo tempo, pois a solicitação de cadastramento pode ser feito, a qualquer momento, no setor de circulação e a divulgação do Boletim é feita em vários canais, tais como, pela página da Biblioteca na Internet, e em vários pontos da Biblioteca.

Há um enorme campo de trabalho para a disseminação de informação científica nas universidades públicas brasileiras, e esse trabalho é urgente, pois as novas Tecnologias da Informação e Comunicação redefiniram a forma de trabalho nas Bibliotecas. Acredita-se que o Boletim Eletrônico da Biblioteca do Instituto de Física, cumpra este papel de disseminador da informação, fazendo com que a Biblioteca busque realizar um trabalho ligado aos interesses da comunidade científica, onde ela participa, interroga e descobre valores, além de poupar o tempo do usuário, através da otimização do serviço. A forma tradicional de disponibilizar produtos e serviços de referencia e informação ainda é largamente difundida, no entanto buscar formas virtuais para 
melhor atender as necessidades de informação do usuário, ainda é a forma dinamicamente mais efetiva de disponibilizar informação. Segundo Rezende (2004) guardar não significa dispor quando se necessita e guardar tem um custo geralmente subestimado quando da criação de sistemas que pretendem ser efetivos. Esta é justamente uma das vantagens competitivas do serviço de referencia virtual, pois as fontes de informação virtuais atualizam-se rapidamente, demandam menor mão de obra e não necessitam de espaço físico para a guarda.

Como reflexão para pesquisas futuras, acredita-se que há indícios fortes de que este trabalho deve ser contínuo, que o Boletim não está totalmente pronto, precisa de ajustes, há também, a necessidade constantes de revisões e atualizações para que a ferramenta esteja sempre em convergência com as necessidades dos usuários que a utilizam.

\section{Referências}

BLATTMANN, U.; SILVA, F. C. C. Colaboração e interação na web 2.0 e biblioteca 2.0. Revista ACB: Biblioteconomia em Santa Catarina, Florianópolis, v. 12, n. 2, p. 191-215, jul./dez. 2007. Disponível em: $<$ http://dialnet.unirioja.es/servlet/fichero articulo?codigo $=2684572>$.

Acesso em: 13 mar. 2014.

BRANDÃO, D. C.; CARVALHO, M. L. N. de. Biblioteca Alberto Nepomuceno da Escola de Música da UFRJ: do raro ao virtual. Disponível em: <http://www.sibi.ufrj.br/bibmusica.pdf>. Acesso em: 4 maio 2009.

CARLSON, S. Survey finds that students use the web but recognize its limitations. Chronicle of Higher Education. July 19, 2002. Disponível em: $<$ http://chronicle.com/article/Survey-Finds-That-Students-/116933/>. Acesso em: 6 maio 2015.

CARVALHO, L. S.; LUCAS, E. R. O. Serviço de referência e informação: do tradicional ao on-line. Disponível em: <http://dici.ibict.br/archive/00000529/01/LidianeElaineServicoReferencia. pdf $>$. Acesso em: 14 fev. 2013.

CUNHA, M. B. da. A biblioteca universitária na encruzilhada. DataGramaZero - Revista de Ciência da Informação, v. 11, n. 6, dez. 2010. Disponível em: <http://dgz.org.br/dez10/Art 07.htm>. Acesso em: 8 maio 2015.

FIGUEIREDO, N. M. de. Paradigmas modernos da ciência da informação. São Paulo: POLIS, 1996.

FIGUEIREDO, N. M. Metodologias para promoção do uso da informação: técnicas aplicadas particularmente em bibliotecas universitárias e especializadas. São Paulo: Nobel, 1991.

GARCEZ, E. M. S.; RADOS, G. J. V. Biblioteca híbrida: um novo enfoque no suporte à educação à distância. Ciência da Informação, Brasília, v. 31, n. 2, p. 44-51, maio/ago. 2002.

GROGAN, D. J. A prática do serviço de referência. Brasília: Briquet de Lemos, 2001. 
INTERNET WORLD STATS. 2010. Disponível em: <http://www.internetworldstats.com/stats.htm>. Acesso em: 4 abr. 2015.

WIKIPÉDIA. Disponível

em: <https://pt.wikipedia.org/wiki/Wikip\%C3\%A9dia:P\%C3\%A1gina_principal $>$. Acesso em: 18 fev. 2015.

LAMB, G. M. How the web changes your reading habits. Christian Science Monitor. June 23, 2005. Disponível em: <http://www.csmonitor.com/2005/0623/p13s02-stin.html >. Acesso em: 10 jul. 2014.

LIMA, D. M. F. de. Escrita colaborativa na biblioteca universitária: o uso do google docs na construção do conhecimento. In: SEMINÁRIO NACIONAL DE BIBLIOTECAS UNIVERSITÁRIAS, 16., 2012, Gramado (RS). Anais... Gramado: $\quad$ UFRS, 2012. Disponível em:< http://www.snbu2012.com.br/anais/pdf/4RC2.pdf>. Acesso em: 13 fev. 2014.

LYNCH, L. M. How to compete: the impact of workplace practices and information technology on productivity. The Review of Economics and Statistics, MIT Press, v. 83, n. 3, p. 434-445, Aug. 2001.

MACEDO, N. D. Princípios e reflexões sobre o serviço de referência e informação. Revista Brasileira de Biblioteconomia e Documentação, São Paulo, v. 23, n. 1, p. 9-37, jan./dez.1990.

MARCONDES, C. H.; MENDONÇA, M. A.; CARVALHO, S. M. Serviços via web em bibliotecas universitárias brasileiras. Perspectivas em Ciência da Informação, Belo Horizonte, v. 11, n. 2, p. 174 -186, maio/ago. 2006.

REZENDE, I. Natura cosméticos: quando é virtuoso ser virtual. Disponível em:

$<$ http://www.ibict.br/cienciadainformacao/include/getdoc. php?id=755\&article=433\&m ode $=$ pdf $>$. Acesso em: 8 out. 2004.

SILVEIRA, A. Marketing em bibliotecas universitárias. Florianópolis: UFSC, 1992.

TARAPANOFF, K.; KLAES, R. R.; CORMIER, P. M. J.

Biblioteca Universitária e contexto acadêmico. In: SEMINÁRIO NACIONAL DE BIBLIOTECAS UNIVERSITÁRIAS, 9.,1996, Curitiba. Anais...Curitiba: FPR; PUC,1996. 